Journal of Applied Analysis

Vol. 5, No. 1 (1999), pp. 125-146

\title{
MULTIVARIABLE REGULAR VARIATION OF FUNCTIONS AND MEASURES
}

\author{
M.M. MEERSCHAERT and H.-P. SCHEFFLER \\ Received November 14, 1996 and, in revised form, December 7, 1998
}

\begin{abstract}
Regular variation is an asymptotic property of functions and measures. The one variable theory is well-established, and has found numerous applications in both pure and applied mathematics. In this paper we present several new results on multivariable regular variation for functions and measures.
\end{abstract}

\section{Introduction}

A Borel measurable function $R: \mathbb{R}^{+} \rightarrow \mathbb{R}^{+}$is said to vary regularly at infinity with index $\rho \in \mathbb{R}$ if, for all $\lambda>0$ we have

$$
\lim _{t \rightarrow \infty} R(\lambda t) / R(x)=\lambda^{\rho} .
$$

A regularly varying function with index zero is also called slowly varying. A comprehensive account of the one variable theory, including a large number of applications, can be found in [4]. The beginnings of a theory of regular variation in $\mathbb{R}^{\urcorner}$can be found in [1], [8], [12], and [22]. The general theory was laid out in [14]. This theory of multivariable regular variation was

1991 Mathematics Subject Classification. 26 B35.

Key words and phrases. Regular variation, weak convergence, Lie groups, multivariate stable distributions.

ISSN 1425-6908 C Heldermann Verlag. 
then generalized to regular varying functions and measures on nilpotent Lie groups in [18].

In this paper we establish some basic results on the geometry of regularly varying functions, measures, and sequences of linear operators on $\mathbb{R}\urcorner$. By geometry we mean that regular variation for linear operators, which is the foundation of the whole theory, leads to a powerful decomposition theorem of the underlying vector space $\mathbb{R}^{\urcorner}$called the spectral decomposition (see Theorem 2.4). Such a spectral decomposition, which was already proved in the slightly different setting of generalized domains of (semi-) attraction of operator (semi-) stable laws, is the key ingredient to various deep theorems on operator (semi-) stable laws. See [15], [17] and the literature cited there.

Then we investigate regularly varying functions on $\mathbb{R}\urcorner$. Our principal result is an extension of Feller's characterization of regularly varying functions (see Theorem 3.14). The theory of regularly varying measures is derived along the same lines as our theory for functions with virtually the same proofs. One might argue that these two theories should be equivalent via a uniqueness and continuity theorem for a certain operator. Unfortunately, as shown in a counter example, this is not the case. Hence we will only sketch the proofs of the theory of regularly varying measures pointing out the differences to the proof in the function case.

Recently, the concept of regular variation on $\mathbb{R}^{\urcorner}$has been generalized to multivariable $\mathrm{R}-\mathrm{O}$ variation, which is the appropriate theory to investigate generalized domains of semistable attraction, see [18]. This theory is again based on the theory of regular variation for linear operators presented here.

\section{Regular variation for linear operators}

Suppose $f: \mathbb{R}^{+} \rightarrow \mathbb{G} \mathbb{L}\left(\mathbb{R}^{\urcorner}\right)$is Borel measurable, where $G L\left(\mathbb{R}^{\urcorner}\right)$is the Lie group of invertible linear operators on $\mathbb{R}\urcorner$. We say that $f$ varies regularly if

$$
\lim _{t \rightarrow \infty} f(\lambda t) f(t)^{-1}=\psi(\lambda) \in G L\left(\mathbb{R}^{\urcorner}\right)
$$

for all $\lambda>0$. In this case, for some linear operator $B$ called the index of $f$ we have $\psi(\lambda)=\lambda^{B}$ for all $\lambda>0$, see [14]. Here $\lambda^{B}=\exp (B \log \lambda)$ where $\exp (A)=I+A+A^{2} / 2 !+A^{3} / 3 !+\cdots$ is the usual exponential operator.

The convergence in (2.1) is automatically uniform on compact subsets of $\lambda>0$, see [1], [14]. A straightforward extension of that proof yields the following technical result, which will be useful in the next section. See also [15], Theorem 3.1.

Definition 2.1. Let $(\mathcal{W},\|\cdot\|)$ be a Banach space and set $S \subset \mathcal{W}$ be closed. For $x \in \mathcal{W}$ we define

$$
\|x-S\|=\inf \{\|x-z\|: z \in S\}
$$


the distance between $x$ and $S$. For a sequence $\left(x_{n}\right)$ in $\mathcal{W}$ we write

$$
x_{n} \rightarrow S \quad \text { if and only if }\left\|x_{n}-S\right\| \rightarrow 0
$$

as $n \rightarrow \infty$.

Lemma 2.2. Suppose $K$ is a compact subset of $G L(\mathbb{R}\urcorner)$ and

$$
f(\lambda t) f(t)^{-1} \rightarrow \lambda^{B} K
$$

as $t \rightarrow \infty$ for all $\lambda>0$. Then this convergence is uniform on compact subsets of $t>0$.

Regular variation on $G L\left(\mathbb{R}^{\urcorner}\right.$) (and more generally on Lie groups) is the most natural extension of the one variable theory. It is also the key to the theory of regularly varying functions and measures, which will be discussed in the following two sections. The main purpose of this section is to establish a structure theorem called the spectral decomposition for regularly varying sequences of linear operators. We begin with a preparatory lemma. If $A_{n} B_{n}^{-1} \rightarrow I$ in $\left.G L(\mathbb{R}\urcorner\right)$ we will write $A_{n} \sim B_{n}$. Suppose $f$ varies regularly with index $B$. Factor the minimal polynomial $g$ of $B$ into $g_{1}(x) \cdots g_{p}(x)$ where all roots of $g_{i}$ have real part $a_{i}$ and $a_{i}<a_{j}$ for $i<j$. Define $V_{i}=\operatorname{Ker}\left(g_{i}(B)\right)$. Then $V_{1} \bigoplus \cdots \bigoplus V_{p}$ is a direct sum decomposition of $\mathbb{R}^{\urcorner}$ into $B$-invariant subspaces, and we may write $B=B_{1} \bigoplus \cdots \bigoplus B_{p}$ where $B_{i}: V_{i} \rightarrow V_{i}$ and every eigenvalue of $B_{i}$ has real part equal to $a_{i}$. We will call this the spectral decomposition of $B$. (This is a special case of the primary decomposition theorem of linear algebra. See for example [5].)

Lemma 2.3. Suppose $f$ varies regularly with index $B$, and let $V_{1}, \ldots, V_{p}$ be the spectral decomposition of $B$. Then for each $i=1, \ldots, p$ there is a subspace $L_{i}$ of $\mathbb{R}^{\urcorner}$such that for $t \rightarrow \infty$

(i) $\operatorname{dim}\left(L_{i}\right)=\operatorname{dim}\left(V_{1} \oplus \cdots \bigoplus V_{i}\right)$;

(ii) $f(t) x /\|f(t) x\| \rightarrow V_{1} \bigoplus \cdots \bigoplus V_{i}$ for all $x \in L_{i}$;

(iii) $f(t) x /\|f(t) x\| \rightarrow V_{i+1} \bigoplus \cdots \bigoplus V_{p}$ for all $x \notin L_{i}$.

(iv) $t^{-\rho}\|f(t) x\| \rightarrow 0$ for all $x \in L_{i}$ and $\rho>a_{i}$.

(v) $t^{-\rho}\|f(t) x\| \rightarrow \infty$ for all $x \notin L_{i}$ and $\rho<a_{i+1}$. 
Proof. Let $V^{\prime}=V_{1} \oplus \cdots \oplus V_{i}$ and $V=V_{i+1} \oplus \cdots \oplus V_{p}$. The subspace $L_{i}$ is in some sense the limit of $f^{-1}(t)\left(V^{\prime}\right)$ as $t \rightarrow \infty$. Fix $\rho \in\left(a_{i}, a_{i+1}\right)$ and note that $t^{-\rho}\left\|t^{B} x\right\| \rightarrow 0$ uniformly on compact subsets of $V^{\prime}$ while $t^{-\rho}\left\|t^{B} x\right\| \rightarrow \infty$ uniformly on compact subsets of $U=\mathbb{R}^{\urcorner}-\mathbb{V}^{\prime}$. For $y \in \mathbb{R}^{\urcorner}$ write $y=r \theta+r^{\prime} \theta^{\prime}$ where $r, r^{\prime} \in \mathbb{R}^{+}$and $\theta, \theta^{\prime}$ are unit vectors in $V, V^{\prime}$ respectively. Given $\delta>0$ define $V_{\delta}=\left\{r \theta+r^{\prime} \theta^{\prime}: r / r^{\prime}>\delta\right\}$, so that $V_{\delta} \subset U$ and $V_{\delta}$ increases to $U$ as $\delta \rightarrow 0$. The regular variation of $f$ implies that $t^{-\rho}\|f(t) x\| \rightarrow \infty$ whenever $x=f^{-1}(t) y$ for any $y \in V_{\delta}$ and $t>t_{0}(\delta)$. Then define

$$
S=\bigcup_{\delta>0} \bigcup_{t>t_{0}(\delta)} f^{-1}(t)\left(V_{\delta}\right)
$$

A computation shows that $t^{-\rho}\|f(t) x\| \rightarrow \infty$ and $f(t) x /\|f(t) x\| \rightarrow V$ for all $x \in S$. Furthermore, if $x \notin S$ then $t^{-\rho}\|f(t) x\| \rightarrow 0$ and $f(t) x /\|f(t) x\| \rightarrow$ $V^{\prime}$. Let $L_{i}=\mathbb{R}^{\urcorner}-\mathbb{S}$. For further details see [17] and [18]. This concludes the proof.

Theorem 2.4 (Spectral decomposition). A Borel measurable function $f$ : $\mathbb{R}^{+} \rightarrow \mathbb{G L}\left(\mathbb{R}^{\urcorner}\right)$varies regularly with index $B$ if and only if $f \sim g T$ for some $T \in G L(\mathbb{R}\urcorner)$ and some $g$ regularly varying with index $B$ such that:

(i) each $V_{i}$ in the spectral decomposition of $B$ is $g$-invariant; and

(ii) $g=g_{1} \bigoplus \cdots \oplus g_{p}$ where each $g_{i}: \mathbb{R}^{+} \rightarrow \mathbb{V}_{\beth}$ is regularly varying with index $B_{i}$.

Proof. Suppose $f$ varies regularly with index $B$. Apply Lemma 2.3 and choose $T \in G L\left(\mathbb{R}^{\urcorner}\right)$so that $L_{i}=T^{-1}\left(V_{1} \bigoplus \cdots \bigoplus V_{i}\right)$ for all $i=1, \ldots, p$. Using the direct sum decomposition, for $x \in V_{i}$ define $g(t) x$ to be the component of $f(t) T^{-1} x$ which lies in $V_{i}$. Extend by linearity. Then use the uniform convergence in (2.1) to show that

$$
\begin{array}{r}
\frac{f(t) T^{-1} \theta}{\left\|f(t) T^{-1} \theta\right\|}-\frac{g(t) \theta}{\|g(t) \theta\|} \rightarrow 0 \\
\frac{\left\|f(t) T^{-1} \theta\right\|}{\|g(t) \theta\|} \rightarrow 1
\end{array}
$$

uniformly on the unit sphere in $V_{i}$ for each $i$. It follows that $f T^{-1} \sim g$. Then it follows immediately from the formula (2.1) that $g$ is also regularly varying with the same index $B$ as $f$. Since each $V_{i}$ is $g$-invariant, each $g_{i}$ varies regularly with index $B_{i}$ by projecting (2.1) onto the subspace $V_{i}$. Conversely, it follows immediately from the formula (2.1) that if $f \sim g T$ and if $g$ varies regularly with index $B$, then so does $f$. This concludes the proof. 


\section{Regular variation for functions}

Let $\Gamma=\mathbb{R}^{\urcorner}-\{\nvdash\}$ and suppose that $F: \Gamma \rightarrow[0, \infty)$ is Borel measurable. We say that $F$ varies regularly if there exist $f: \mathbb{R}^{+} \rightarrow \mathbb{G} \mathbb{L}\left(\mathbb{R}^{\urcorner}\right)$and $R$ : $\mathbb{R}^{+} \rightarrow \mathbb{R}^{+}$, both regularly varying, $R$ not slowly varying, such that

$$
\lim _{t \rightarrow \infty} F\left(f(t)^{-1} x_{t}\right) / R(t)=\varphi(x)>0
$$

whenever $x_{t} \rightarrow x$ in $\Gamma$. In this case the limit function $\varphi$ satisfies

$$
t \varphi(x)=\varphi\left(t^{-E} x\right)
$$

for all $t>0$ and all $x \in \Gamma$, where $E=\beta^{-1} B$ and $\beta, B$ denote the index of $R$ and $f$, respectively, see [14]. We say that $F$ varies regularly with index $E$. If all eigenvalues of $B$ have positive (negative) real part, then Lemma 2.3 shows that $f(t)^{-1} x$ tends to zero (infinity) in norm as $t \rightarrow 0$, and we say that $F$ varies regularly at zero (infinity), respectively. In this section we investigate the structure of regularly varying functions.

Remark 3.1. The definition of a regularly varying function $F$ can be rewritten in the following way: Let $f: \mathbb{R}^{+} \rightarrow \mathbb{G} \mathbb{L}\left(\mathbb{R}^{\urcorner}\right)$be regularly varying with index $B$ and let $R: \mathbb{R}^{+} \rightarrow \mathbb{R}^{+}$be regularly varying with index $\beta \neq 0$. Then $\lim _{t \rightarrow \infty} F\left(f(t)^{-1} x_{t}\right) / R(t)=\varphi(x)$. We have to consider two cases separately.

(a) The case $\beta>0$ : Then $R(t) \rightarrow \infty$ as $t \rightarrow \infty$. Choose an asymptotic inverse $S \in \mathrm{RV}(1 / \beta)$ such that $R \circ S(t) \sim t$ (see [20] Theorem on page $21)$. Here and in the following let us agree to write $f \sim g$ for functions $f, g: \mathbb{R}^{+} \rightarrow \mathbb{R}^{+}$if $f(t) / g(t) \rightarrow 1$ as $t \rightarrow \infty$. Define $f_{1}(t)=f(S(t))$. Then $f_{1}$ is regularly varying with index $E=(1 / \beta) B$ and it follows that

$$
\lim _{t \rightarrow \infty} \frac{F\left(f_{1}(t)^{-1} x_{t}\right)}{t}=\varphi(x)
$$

whenever $x_{t} \rightarrow x$ in $\Gamma$.

(b) The case $\beta<0$ : Then $R(t) \rightarrow 0$ as $t \rightarrow \infty$ and $1 / R \in \operatorname{RV}(-\beta)$. Choose an asymptotic inverse $S \in \mathrm{RV}(-1 / \beta)$ such that $1 / R \circ S(t) \sim t$. Define $f_{1}(t)=f(S(t))$. Then $f_{1}$ is regularly varying with index $(-1 / \beta) B$ and it follows that

$$
\lim _{t \rightarrow \infty} t F\left(f_{1}(t)^{-1} x_{t}\right)=\varphi(x)
$$

whenever $x_{t} \rightarrow x$ in $\Gamma$.

We begin with an examination of the limit function. The following is not the most general treatment possible, but it is sufficient for our purposes.

Definition 3.2. A function $\varphi: \Gamma \rightarrow(0, \infty)$ is called admissible if it is continuous and if either 
(i) $\varphi\left(x_{t}\right)+\varphi\left(-x_{t}\right) \rightarrow \infty$ whenever $\left\|x_{t}\right\| \rightarrow \infty$ or

(ii) $\varphi\left(x_{t}\right)+\varphi\left(-x_{t}\right) \rightarrow 0$ whenever $\left\|x_{t}\right\| \rightarrow \infty$.

Since in (3.1) there are only nonsingular operators allowed we restrict ourselves to operators from $G L\left(\mathbb{R}^{\urcorner}\right)$in the following theory. (It is possible to allow all linear operators $A$ in the following definition but then we need the additional property $\varphi(0)=0$ of an admissible function.)

Definition 3.3. An operator $A \in G L\left(\mathbb{R}^{\urcorner}\right)$is called a symmetry of a function $\varphi: \Gamma \rightarrow \mathbb{R}^{+}$if $\varphi(A x)=\varphi(x)$ for all $x \in \Gamma$. Let

$$
\mathcal{S}(\varphi)=\{A \in G L(\mathbb{R}\urcorner): \mathbb{A} \text { is a symmetry of } \varphi\} .
$$

Lemma 3.4. For a function $\varphi: \Gamma \rightarrow(0, \infty)$ define $g(x)=\varphi(x)+\varphi(-x)$. Then $\mathcal{S}(\varphi) \subset \mathcal{S}(g)$.

Proof. Assume $\varphi \in \mathcal{S}(\varphi)$. Then $\varphi(A x)=\varphi(x)$ for all $x \in \Gamma$. Especially $\varphi(A(-x))=\varphi(-A x)=\varphi(-x)$ for all $x \in \Gamma$. Hence $g(A x)=\varphi(A x)+$ $\varphi(-A x)=\varphi(x)+\varphi(-x)=g(x)$ for all $x \in \Gamma$ so $A \in \mathcal{S}(g)$. This concludes the proof.

Theorem 3.5. The symmetries $\mathcal{S}(\varphi)$ of an admissible function $\varphi$ form a compact subgroup of $G L(\mathbb{R}\urcorner)$.

Proof. Obviously $\mathcal{S}(\varphi)$ is closed under composition and inverses, so $\mathcal{S}(\varphi)$ is a subgroup of $G L\left(\mathbb{R}^{\urcorner}\right)$. If $A_{n} \in \mathcal{S}(\varphi)$ and $A_{n} \rightarrow A$ then for all $x \in \Gamma$ we have $\varphi(x)=\varphi\left(A_{n} x\right) \rightarrow \varphi(A x)$ which implies that $\varphi(A x)=\varphi(x)$, and so $\mathcal{S}(\varphi)$ is closed. Finally, if $A_{n} \in \mathcal{S}(\varphi)$ then by Lemma $3.4 A_{n} \in \mathcal{S}(g)$, where $g(x)=\varphi(x)+\varphi(-x)$ as before. Then $g(x)=g\left(A_{n} x\right)$ implies that $\left\|A_{n} x\right\|$ is bounded (otherwise $g\left(A_{n} x\right)$ tends to either zero or infinity), and so $\mathcal{S}(g)$ is bounded and hence $\mathcal{S}(\varphi)$ is compact. This concludes the proof.

In the following we use the notation $A F(x)=F\left(A^{-1} x\right)$ for $A \in G L\left(\mathbb{R}^{\urcorner}\right)$ and $F: \Gamma \rightarrow \mathbb{R}^{+}$.

Definition 3.6. Let $\mathcal{F}$ denote the set of all Borel measurable functions $F: \Gamma \rightarrow \mathbb{R}^{+}$which are monotone on rays, by which we mean that $t \mapsto F(t x)$ is a monotone function of $t>0$ for all $x \in \Gamma$. For $F_{n} \in \mathcal{F}$ and an admissible function $\varphi: \Gamma \rightarrow(0, \infty)$ we write $F_{n} \rightarrow \varphi$ if $F_{n}\left(x_{n}\right) \rightarrow \varphi(x)$ whenever $x_{n} \rightarrow x$ in $\Gamma$. 
The next step is to establish a convergence of types theorem for real-valued functions on $\mathbb{R}\urcorner$. We begin with two simple lemmas.

Lemma 3.7. Let $F_{n} \in \mathcal{F}, \varphi$ admissible and $A_{n} \in G L\left(\mathbb{R}^{\urcorner}\right)$. If $F_{n} \rightarrow \varphi$ then $A_{n} F_{n} \rightarrow \varphi$ if and only if $A_{n} \rightarrow \mathcal{S}(\varphi)$.

Proof. Let $\rho(x)=\varphi(x)+\varphi(-x)$ and $G_{n}(x)=F_{n}(x)+F_{n}(-x)$. If $\varphi$ satisfies (ii) of Definition 3.2 then $\sup _{\|x\|=r} \rho(x) \rightarrow 0$ as $r \rightarrow \infty$. Furthermore, since $F_{n} \in \mathcal{F}$ and $F_{n} \rightarrow \varphi$ uniformly over any fixed sphere, it follows that $t \mapsto F_{n}(t x)$ is monotone decreasing for all $x \in \Gamma$ and hence $t \mapsto G_{n}(t x)$ is monotone decreasing for all $x \in \Gamma$ too. Then the uniform convergence of $F_{n} \rightarrow \varphi$ over any fixed sphere implies that $G_{n} \rightarrow \rho$ uniformly on compact subsets of $\Gamma$ and hence $G_{n}\left(x_{n}\right) \rightarrow 0$ whenever $\left\|x_{n}\right\| \rightarrow \infty$. Similarly if $\varphi$ satisfies (i) in Definition 3.2 then $G_{n}\left(x_{n}\right) \rightarrow \infty$ whenever $\left\|x_{n}\right\| \rightarrow \infty$. Now if $A_{n} F_{n} \rightarrow \varphi$ then for all $x \in \Gamma$ we have $F_{n}\left(A_{n}^{-1} x\right) \rightarrow \varphi(x)>0$ and $F_{n}\left(A_{n}^{-1}(-x)\right) \rightarrow \varphi(-x)>0$. Then $G_{n}\left(A_{n}^{-1} x\right) \rightarrow \rho(x)>0$ for all $x \in \Gamma$. If $\left\|A_{n}^{-1} x\right\| \rightarrow \infty$ then $G_{n}\left(A_{n}^{-1} x\right)$ tends to either zero or infinity, which is a contradiction. Thus the sequence $A_{n}^{-1}$ is relatively compact. If along a subsequence $n^{\prime}$ we have $A_{n^{\prime}}^{-1} \rightarrow C$ then $F_{n}\left(A_{n^{\prime}}^{-1} x\right) \rightarrow \varphi(C x)$ and so $C \in \mathcal{S}(\varphi)$. Since $\mathcal{S}(\varphi)$ is a compact group, taking inverses yields $A_{n} \rightarrow \mathcal{S}(\varphi)$. Conversely, if $A_{n} \rightarrow \mathcal{S}(\varphi)$ then for any subsequence there is a further subsequence $n^{\prime}$ such that $A_{n^{\prime}} \rightarrow A$ for some $A \in \mathcal{S}(\varphi)$. Then $A_{n^{\prime}} F_{n^{\prime}} \rightarrow A \varphi=\varphi$. It follows that $A_{n} F_{n} \rightarrow \varphi$. This concludes the proof.

Lemma 3.8. Let $F_{n} \in \mathcal{F}, \varphi, \varphi_{0}$ admissible and $\left.A_{n} \in G L(\mathbb{R}\urcorner\right)$. If $F_{n} \rightarrow \varphi$ and $A_{n} F_{n} \rightarrow \varphi_{0}$ then $\varphi_{0}=C \varphi$ for some linear operator $\left.C \in G L(\mathbb{R}\urcorner\right)$.

Proof. Let $\rho$ and $G_{n}$ be as in the proof of Lemma 3.7. If $A_{n} F_{n} \rightarrow \varphi_{0}$ then for all $x \in \Gamma$ we have $F_{n}\left(A_{n}^{-1} x\right) \rightarrow \varphi_{0}(x)>0$. If $\left\|A_{n}^{-1} x\right\| \rightarrow \infty$ then the fact that $F_{n} \rightarrow \varphi$ admissible implies that $G_{n}\left(A_{n}^{-1} x\right)$ tends to either zero or infinity, which is a contradiction (see the proof of Lemma 3.7). Then the sequence $A_{n}^{-1}$ is relatively compact. If along a subsequence $n^{\prime}$ we have $A_{n^{\prime}}^{-1} \rightarrow C_{0}$ then $F_{n^{\prime}}\left(A_{n^{\prime}}^{-1} x\right) \rightarrow \varphi_{0}(x)=\varphi\left(C_{0} x\right)$ for all $x \in \Gamma$. Since $\varphi_{0}$ is admissible we cannot have $C_{0} x=0$ for any $x \in \Gamma$, so $C=C_{0}^{-1}$ exists and we may write $\varphi_{0}=C \varphi$. This concludes the proof.

Theorem 3.9 (Convergence of types). Let $F_{n} \in \mathcal{F}, \varphi, \varphi_{0}$ admissible and $A_{n}, B_{n} \in G L\left(\mathbb{R}^{\urcorner}\right)$. Suppose $B_{n} F_{n} \rightarrow \varphi$. Then $A_{n} F_{n} \rightarrow \varphi_{0}$ if and only if $\varphi_{0}=C \varphi$ and $A_{n} B_{n}^{-1} \rightarrow C \mathcal{S}(\varphi)$ for some $C \in G L\left(\mathbb{R}^{\urcorner}\right)$. 
Proof. If $A_{n} F_{n} \rightarrow \varphi_{0}$ then $\left(A_{n} B_{n}^{-1}\right) B_{n} F_{n} \rightarrow \varphi_{0}$ and so Lemma 3.8 yields $\varphi_{0}=C \varphi$. Then $C^{-1}\left(A_{n} B_{n}^{-1}\right) B_{n} F_{n} \rightarrow \varphi$ and so Lemma 3.7 yields $C^{-1}\left(A_{n} B_{n}^{-1}\right)$ $\rightarrow \mathcal{S}(\varphi)$. Multiply both sides by $C$ on the left. Conversely, if $A_{n} B_{n}^{-1} \rightarrow$ $C \mathcal{S}(\varphi)$ then $C^{-1}\left(A_{n} B_{n}^{-1}\right) \rightarrow \mathcal{S}(\varphi)$ and so Lemma 3.7 yields $C^{-1}\left(A_{n} B_{n}^{-1}\right) B_{n} F_{n}$ $\rightarrow \varphi$. Simplify and then multiply both sides by $C$ on the left to obtain $A_{n} F_{n} \rightarrow \varphi_{0}$ which concludes the proof.

Now we come to the main point of this section, which is to establish the basic characterization of regularly varying functions which are monotone on rays. We begin with two technical results.

Theorem 3.10. Let $F \in \mathcal{F}, \varphi$ admissible and $\left.A_{n} \in G L(\mathbb{R}\urcorner\right)$. Suppose $a_{n}>0$ is a regularly varying sequence with index $\beta \neq 0$ and that $a_{n}\left(A_{n} F\right) \rightarrow$ $\varphi$. Then there exists a linear operator $B$ such that for all $t>0: t^{\beta} \cdot \varphi=t^{B} \varphi$; and

$$
A_{[t n]} A_{n}^{-1} \rightarrow t^{-B} \mathcal{S}(\varphi)
$$

Proof. Let $F_{n}=a_{n} \cdot F$ so that $A_{n} F_{n} \rightarrow \varphi$. Regular variation of the sequence $a_{n}$ implies that $A_{[t n]} F_{n}=\left(a_{[t n]} a_{n}^{-1}\right)^{-1} A_{[t n]} F_{[t n]} \rightarrow t^{-\beta} \cdot \varphi$. Then Theorem 3.9 yields $t^{-\beta} \cdot \varphi=C_{t} \varphi$ and $A_{[t n]} A_{n}^{-1} \rightarrow C_{t} \mathcal{S}(\varphi)$ for all $t>0$, where $C_{t} \in G L\left(\mathbb{R}^{\urcorner}\right)$. Define $G_{t}=C_{t} \mathcal{S}(\varphi)$ and note that $C \varphi=t^{-\beta} \cdot \varphi$ if and only if $C=C_{t} A$ for some $A \in \mathcal{S}(\varphi)$. Define $G$ to be the union of all $G_{t}$ taken over $t>0$. If $C \in G_{t}$ then $C \varphi=t^{-\beta} \cdot \varphi$ which implies $C^{-1} \varphi=t^{\beta} \cdot \varphi$ and so $C^{-1} \in G_{1 / t}$. If $C \in G_{t}$ and $D \in G_{s}$ then $C D \varphi=t^{-\beta} s^{-\beta} \cdot \varphi$ implies that $C D \in G_{s t}$. Therefore $G_{t}^{-1} \subset G_{1 / t}$ and $G_{s} G_{t} \subset G_{s t}$. Now replace $t$ by $1 / t$ in the first inclusion and replace $s$ and $t$ by $1 / s$ and $s t$ in the second to obtain equality. Then $G$ is a group. If $D_{n} \in G$ and $D_{n} \rightarrow D$ in $G L\left(\mathbb{R}^{\urcorner}\right)$ write $D_{n}=C_{t_{n}} A_{n}$ for some $t_{n}>0$ and $A_{n} \in \mathcal{S}(\varphi)$. Since $D_{n} \varphi \rightarrow D \varphi$ we must have $t_{n}$ relatively compact in $\mathbb{R}^{+}$, and it is immediate from Theorem 3.5 that $G_{t}$ is compact. If along a subsequence $t_{n^{\prime}} \rightarrow t$ and $A_{n^{\prime}} \rightarrow A$ then $D_{n^{\prime}} \varphi=C_{t_{n^{\prime}}} A_{n^{\prime}} \varphi \rightarrow t^{-\beta} \cdot \varphi$ which yields $D=C_{t} A \in G_{t}$. Thus $G$ is closed in $G L\left(\mathbb{R}^{\urcorner}\right)$. Define $\zeta(C)=t$ for all $C \in G_{t}$ to obtain a continuous group homomorphism $\zeta: G \rightarrow \mathbb{R}^{+}$and note that since $G_{t} \cap G_{s}=\emptyset$ for $t \neq s$ this homomorphism is well defined. Since $G$ is a Lie group, the connected component of the identity $G_{0}$ is an open normal subgroup which consists of one parameter semigroups $\left\{t^{E}: t>0\right\}$ for some elements $E$ of the Lie algebra of $G$. It is easy to check that $\zeta$ is open, and so $\zeta\left(G_{0}\right)$ is an open subgroup of $\mathbb{R}^{+}$and hence must be $\mathbb{R}^{+}$. Then there must exist a linear operator $B$ such that $\zeta\left(t^{-B}\right)=t$ for all $t>0$. Then $t^{-B} \in G_{t}$ and so we may write $G_{t}=t^{-B} \mathcal{S}(\varphi)$ for all $t>0$. This concludes the proof. 
In the case of Theorem 3.10, if we define $E=\beta^{-1} B$ then (3.2) holds for all $t>0$ and all $x \in \Gamma$. In other words, we have $t \varphi=t^{E} \varphi$ for all $t>0$.

Definition 3.11. Let $\varphi$ be admissible. Then any linear operator $E$ such that

$$
t \cdot \varphi=t^{E} \varphi
$$

holds for all $t>0$ is called an exponent of $\varphi$. Let

$$
\mathcal{E}(\varphi)=\{E: E \text { is an exponent of } \varphi\} .
$$

Exponents in general are not unique. The next theorem describes the structure of the set $\mathcal{E}(\varphi)$ of exponents of an admissible function $\varphi$.

Theorem 3.12. Exponents and symmetries of an admissible function $\varphi$ are related by

$$
\mathcal{E}(\varphi)=E+T \mathcal{S}(\varphi)
$$

where $E \in \mathcal{E}(\varphi)$ is arbitrary and $T \mathcal{S}(\varphi)$ denotes the tangent space. One can always select an exponent $E_{0}$ which commutes with every symmetry $A \in \mathcal{S}(\varphi)$.

Proof. Let $G$ and $\zeta$ be as in the proof of Theorem 3.10. Define a continuous group homomorphism $L: T G \rightarrow \mathbb{R}$ by letting $L(X)=\log \zeta(\exp (X))$. Then $T \mathcal{S}(\varphi)=\operatorname{ker} L$ and $E \in \mathcal{E}(\varphi)$ if and only if $E \in T G$ and $L(E)=-1 / \beta$. Use continuity to check that $L(t X)=t L(X)$, and then take the derivative of $L(t X)+L(t Y)$ at $t=0$ to show that $L$ is actually a linear functional. Equation (3.4) follows. Finally if $E \in \mathcal{E}(\varphi)$ is arbitrary we may define

$$
E_{0}=\int_{A \in \mathcal{S}(\varphi)} A E A^{-1} \mathcal{H}(d A)
$$

where $\mathcal{H}$ is Haar measure on the compact group $\mathcal{S}(\varphi)$. It is easy to check that $E_{0}=A E_{0} A^{-1}$ for every $A \in \mathcal{S}(\varphi)$, and since (3.4) holds we know that $\mathcal{E}(\varphi)$ is closed and convex, so $E_{0} \in \mathcal{E}(\varphi)$. This concludes the proof.

Remark 3.13. If $B$ is the linear operator in Theorem 3.10 then we know that $E=\beta^{-1} B$ is an element of $\mathcal{E}(\varphi)$. Suppose $E^{\prime} \in \mathcal{E}(\varphi)$ is another exponent and let $B^{\prime}=\beta E^{\prime}$. Then Theorem 3.12 implies that $E^{\prime}=E+X$ where $X \in T \mathcal{S}(\varphi)$. Using the fact that $t^{X} \in \mathcal{S}(\varphi)$, it is easy to see that $t^{-B^{\prime}} \mathcal{S}(\varphi)=t^{-B} \mathcal{S}(\varphi)$ for all $t>0$. Then we may assume that the linear operator $B$ in (3.3) commutes with every symmetry.

The next result is our basic characterization of regularly varying functions which are monotone on rays. The one variable version is due to Feller [6]. 
Theorem 3.14. Let $F \in \mathcal{F}, \varphi$ admissible and $A_{n} \in G L\left(\mathbb{R}^{\urcorner}\right)$. Suppose $a_{n}>0$ is a regularly varying sequence with index $\beta \neq 0$ and that $a_{n} A_{n} F \rightarrow$ $\varphi$. Then $F$ is regularly varying with index $E$ for any $E \in \mathcal{E}(\varphi)$.

Proof. Theorem 3.10 implies that (3.3) holds for some linear operator $B$, and Lemma 2.2 shows that this convergence is uniform on compact subsets of $t>0$. Remark 3.13 shows that we may take $B=\beta E$ where $E \in \mathcal{E}(\varphi)$ is arbitrary. To begin with, we will assume that $E$ is commuting, so that $t^{-B} \mathcal{S}(\varphi)=\mathcal{S}(\varphi) t^{-B}$ for all $t>0$. Now suppose that $G_{n} \in \mathcal{S}(\varphi)$ and let $B_{n}=G_{n} A_{n}$. Then Lemma 3.7 shows that $a_{n} B_{n} F \rightarrow \varphi$ too. In order to show that $F$ varies regularly with index $E$, it will suffice to show that we can choose $G_{n}$ to make $B_{n}$ vary regularly with index $-B$. Since the argument is quite similar to the proof of the theorem in [14] we only sketch the argument here. Since $\mathcal{S}(\varphi)$ is a compact subgroup of $G L(\mathbb{R}\urcorner)$, there exists an inner product on $\mathbb{R}^{\urcorner}$which makes every symmetry $G \in \mathcal{S}(\varphi)$ orthogonal. We will use the norm associated with this inner product to define the sequence $B_{n}$. Let $B_{1}=A_{1}$. For all other $n$, suppose that $2^{k}<n \leq 2^{k+1}$, and let $r=n / 2^{k}$. Select $G_{n} \in \mathcal{S}(\varphi)$ to minimize $\left\|A_{n} B_{2^{k}}^{-1}-r^{-\bar{B}} \mathcal{S}(\varphi)\right\|$. Using the uniform convergence and the fact that $B$ is commuting, a computation shows that $B_{[t n]} B_{n}^{-1} \rightarrow t^{-B}$ for all $t>0$. Finally suppose that $E \in \mathcal{E}(\varphi)$ is not commuting, and use Theorem 3.12 to write $E=E_{0}+X$ where $E_{0} \in \mathcal{E}(\varphi)$ is commuting and $X \in T \mathcal{S}(\varphi)$. Define $B=\beta^{-1} E$ and $B_{0}=\beta^{-1} E_{0}$. The above argument yields a sequence $B_{n}$ such that $a_{n} B_{n} F \rightarrow \varphi$ and $B_{n}$ varies regularly with index $-B_{0}$. Let $C_{n}=n^{-X} B_{n}$ and notice that $n^{-X} \in \mathcal{S}(\varphi)$ for all $n$. Then Lemma 3.7 implies that $a_{n} C_{n} F \rightarrow \varphi$, and since $B_{0}$ is commuting, it is easy to check that $C_{n}$ varies regularly with index $-B$. This concludes the proof.

Remark 3.15. In order to apply Theorem 3.14 one must verify that the limit function $\varphi$ is admissible. In fact if $a_{n} F\left(A_{n}^{-1} x_{n}\right) \rightarrow \varphi(x)>0$ whenever $x_{n} \rightarrow x$ in $\Gamma$ then $\varphi$ must be continuous. Suppose not. Then for some $\varepsilon>0$ and some $y_{k} \rightarrow x$ in $\Gamma$ we have $\left|\varphi\left(y_{k}\right)-\varphi(x)\right|>\varepsilon$ for all $k$. We also have for each fixed $k$ that $a_{n} F\left(A_{n}^{-1} y_{k}\right) \rightarrow \varphi\left(y_{k}\right)$ as $n \rightarrow \infty$, so that for each $k$ there exists an integer $n_{k}$ such that $\left|a_{n} F\left(A_{n}^{-1} y_{k}\right)-\varphi\left(y_{k}\right)\right|<\varepsilon / 2$ for all $n \geq n_{k}$. Without loss of generality we can choose $n_{k}$ monotone increasing. Now let $x_{n}=y_{n_{k}}$ for all $n_{k-1}<n \leq n_{k}$. Then for all $n$ we have

$$
\left|a_{n} F\left(A_{n}^{-1} x_{n}\right)-\varphi(x)\right| \geq\left|\varphi\left(x_{n}\right)-\varphi(x)\right|-\left|a_{n} F\left(A_{n}^{-1} x_{n}\right)-\varphi\left(x_{n}\right)\right| \geq \varepsilon / 2
$$

which is a contradiction. A similar argument shows that if $F$ varies regularly and (3.1) holds then $\varphi$ is continuous. Furthermore, if $F$ varies regularly at zero or infinity then $\varphi$ is admissible. For example, if every eigenvalue of $E$ has positive real part and $\left\|x_{t}\right\| \rightarrow \infty$, write $x_{t}=\left(r_{t}\right)^{-E} \theta_{t}$ where $r_{t}>0$ and 
$\left\|\theta_{t}\right\|=1$. Then (3.2) yields $\varphi\left(x_{t}\right)+\varphi\left(-x_{t}\right)=r_{t}\left(\varphi\left(\theta_{t}\right)+\varphi\left(-\theta_{t}\right)\right) \rightarrow 0$ as $t \rightarrow 0$ since $r_{t} \rightarrow 0$ and $\varphi$ is bounded on the unit sphere.

\section{Regular variation for measures}

In this section we develop a multivariable theory of regularly varying measures.

Let $\mathcal{M}$ denote the set of $\sigma$ finite Borel measures on $\Gamma$ which are finite outside every neighborhood of the origin with the topology of weak convergence. In this topology we have $\mu_{n} \rightarrow \mu$ if and only if $\mu_{n}(S) \rightarrow \mu(S)$ for all Borel subsets $S \subset \Gamma$ which are bounded away from the origin and whose topological boundary $\partial S$ has $\mu$-measure zero.

Definition 4.1. A measure $\mu \in \mathcal{M}$ varies regularly if there exist $f: \mathbb{R}^{+} \rightarrow$ $\mathbb{G L}\left(\mathbb{R}^{\urcorner}\right)$and $R: \mathbb{R}^{+} \rightarrow \mathbb{R}^{+}$, both regularly varying, $R$ not slowly varying, such that

$$
\frac{\mu\left\{f^{-1}(t) d x\right\}}{R(t)} \rightarrow \phi\{d x\}
$$

as $t \rightarrow \infty$ for some $\phi \in \mathcal{M}$ which is full, by which we mean that $\phi$ cannot be supported on any $k-1$ dimensional linear subspace.

If (4.1) holds then the limit measure $\phi$ satisfies

$$
t \cdot \phi\{d x\}=\phi\left\{t^{-E} d x\right\}
$$

for all $t>0$, where $E=\beta^{-1} B$ and $\beta, B$ denote the index of $R$ and $f$, respectively, see [14]. We say that $\mu$ varies regularly with index $E$.

Remark 4.2 (see Remark 3.1). As in the function case we have the following alternative definition of regularly varying measures: Let $R: \mathbb{R}^{+} \rightarrow \mathbb{R}^{+}$ be regularly varying with index $\beta \neq 0$ and $f: \mathbb{R}^{+} \rightarrow \mathbb{G} \mathbb{L}\left(\mathbb{R}^{\urcorner}\right)$be regularly varying with index $B$.

(a) The case $\beta>0$ :

$$
\lim _{t \rightarrow \infty} \frac{\mu\left\{f_{1}(t)^{-1} d x\right\}}{t}=\phi\{d x\}
$$

where $f_{1}$ is regularly varying with index $(1 / \beta) B$.

(b) The case $\beta<0$ :

$$
\lim _{t \rightarrow \infty} t \mu\left\{f_{1}(t)^{-1} d x\right\}=\phi\{d x\}
$$

where $f_{1}$ is regularly varying with index $-(1 / \beta) B$. 
We first investigate the possible limit measures in (4.1). In contrast to the case of regularly varying functions considered above it follows that only certain $E$ in (4.2) are allowed.

Remark 4.3. If $\phi \in \mathcal{M}$ is full and satisfies (4.2) then the real parts of the eigenvalues of $E$ in (4.2) are necessarily non-negative. In fact assume that $a_{1}<\cdots<a_{p}$ are the real parts of the eigenvalues of $E$ and let $\left.\mathbb{R}\right\urcorner=$ $\mathbb{V}_{\nVdash} \oplus \cdots \oplus \mathbb{V}$, be the direct sum decomposition into $E$-invariant subspaces as in section 2. Now assume that $a_{1}<0$. Let $\theta_{0} \in V_{1}^{*}$ be a unit vector, where $V_{1}^{*}$ denotes the dual space of $V_{1}$. Then (4.2) implies

$$
t \cdot \phi\left\{x:\left|\left\langle x, \theta_{0}\right\rangle\right|>R\right\}=\phi\left\{x:\left|\left\langle x, t^{E^{*}} \theta_{0}\right\rangle\right|>R\right\}
$$

for all $R>0$ and $t>0$. Write $t^{E^{*}} \theta_{0}=r_{t} \theta_{t}$ for some $\left\|\theta_{t}\right\|=1$ and $r_{t}>0$ and note that since $a_{1}<0$ and $\theta_{0} \in V_{1}^{*}$ we have $r_{t} \rightarrow 0$ as $t \rightarrow \infty$. Furthermore $\phi\left\{x:\left|\left\langle x, \theta_{0}\right\rangle\right|>R\right\} \leq \phi\{x:\|x\|>R\}<\infty$. But $\phi\left\{x:\left|\left\langle x, t^{E^{*}} \theta_{0}\right\rangle\right|>R\right\}=$ $\phi\left\{x:\left|\left\langle x, \theta_{t}\right\rangle\right|>R / r_{t}\right\} \rightarrow 0$ as $t \rightarrow \infty$. Hence the right hand side of (4.3) tends to zero whereas the left hand side of (4.3) either tends to infinity as $t \rightarrow \infty$ which is a contradiction or $\phi\left\{x:\left|\left\langle x, \theta_{0}\right\rangle\right|>R\right\}=0$. Since $R>0$ is arbitrary, we have $\phi\left\{x:\left|\left\langle x, \theta_{0}\right\rangle\right| \neq 0\right\}=0$ which contradicts the assumption that $\phi$ is full.

In the following we assume that the real parts of the eigenvalues of $E$ in (4.2) are positive.

Next we define our class of admissible measures which will contain the possible limit measures in our theory of regularly varying measures. We will show later that full measures $\phi$ satisfying (4.2) are in fact admissible.

Definition 4.4. A measure $\phi \in \mathcal{M}$ is admissible if $\phi\{y:\langle x, y\rangle=1\}=0$ and $\phi\{y:|\langle x, y\rangle|>1\}>0$ for all $x \in \Gamma$, and $\phi(\Gamma)=\infty$.

Remark 4.5. The condition $\phi\{y:\langle x, y\rangle=1\}=0$ for all $x \in \Gamma$ is the substitute for the continuity of an admissible function (see Definition 3.2). Furthermore $\phi\{y:|\langle x, y\rangle|>1\}>0$ corresponds to the condition that $\varphi>0$ and $\phi(\Gamma)=\infty$ corresponds to condition (i) of Definition 3.2.

Before we can show that measures $\phi$ satisfying (4.2) are admissible we need a structural theorem showing that any measure $\phi$ in (4.2) can be desintegrated according to the orbits of the one-parameter group $\left(t^{E}\right)_{t>0}$.

Theorem 4.6. Let $\phi \in \mathcal{M}$ satisfy (4.2) for some index $E$ with positive real parts of the eigenvalues. Then there exists a norm $\|\cdot\|_{0}$ on $\mathbb{R}^{\urcorner}$such that for all Borel subsets $A \subset \Gamma$ we have

$$
\phi(A)=\int_{S} \int_{0}^{\infty} 1_{A}\left(t^{E} \theta\right) \frac{d t}{t^{2}} d \lambda(\theta)
$$


where $\lambda$ is a finite Borel measure on the unit sphere $S=\left\{x:\|x\|_{0}=1\right\}$ with respect to $\|\cdot\|_{0}$.

Proof. Let $\|\cdot\|$ denote the Euclidean norm on $\mathbb{R}^{\urcorner}$and define

$$
\|x\|_{0}=\int_{0}^{1}\left\|t^{E} x\right\| \frac{d t}{t} .
$$

Since the real parts of the eigenvalues of $E$ are positive we get, following [9] p.136, that there exists a $\delta>0$ and a $C>0$ such that $\left\|t^{E} x\right\| \leq C t^{\delta}\|x\|$ for all $x \in \mathbb{R}^{\urcorner}$and all $0 \leq t \leq 1$. Hence $\|\cdot\|_{0}$ is well defined and it is easy to see that it is a norm on $\mathbb{R}\urcorner$. It follows from [13] that for all $x \neq 0$ the mapping $t \mapsto\left\|t^{E} x\right\|_{0}$ is strictly increasing and that $\psi:(0, \infty) \times S \rightarrow \Gamma, \psi(t, \theta)=t^{E} \theta$ is a homeomorphism, where $S=\left\{x \in \mathbb{R}^{\urcorner}:\|\curvearrowleft\|_{\nvdash}=\nVdash\right\}$.

For a Borel subset $F \subset S$ we define

$$
\lambda(F)=\phi\left\{t^{E} \theta: t \geq 1, \theta \in F\right\} .
$$

Then $\lambda$ is a finite Borel measure on $S$. For $s>0$ and a Borel subset $F \subset S$ let

$$
A=\left\{t^{E} \theta: t \geq s, \theta \in F\right\} .
$$

Then we get from (4.2) and the definition of $\lambda$

$$
\begin{aligned}
\int_{S} \int_{0}^{\infty} 1_{A}\left(r^{E} \theta\right) \frac{d r}{r^{2}} d \lambda(\theta) & =\int_{F} \int_{s}^{\infty} \frac{d r}{r^{2}} d \lambda(\theta) \\
& =s^{-1} \lambda(F) \\
& =s^{-1} \phi\left\{t^{E} \theta: t \geq 1, \theta \in F\right\} \\
& =\phi\left(s^{E}\left\{t^{E} \theta: t \geq 1, \theta \in F\right\}\right. \\
& =\phi\left\{t^{E} \theta: t \geq s, \theta \in F\right\}=\phi(A) .
\end{aligned}
$$

But the sets of the form $A$ in (4.5) form a $\cap$-stable generator of the Borel $\sigma$-algebra of $\Gamma$ and hence (4.4) holds for all Borel subsets $A \subset \Gamma$. This concludes the proof.

Our next result shows that measures $\phi$ satisfying the transformation formula (4.2) are admissible.

Theorem 4.7. Let $\phi \in \mathcal{M}$ be full and satisfies (4.2) for some index $E$ with positive real parts of the eigenvalues. Then $\phi$ is admissible.

Proof. For $x \in \Gamma$ we get from Theorem 4.6 that

$$
\phi\{y:\langle x, y\rangle=1\}=\int_{S} \int_{0}^{\infty} 1_{\{y:\langle x, y\rangle=1\}}\left(t^{E} \theta\right) \frac{d t}{t^{2}} d \lambda(\theta) .
$$


But since the real parts of the eigenvalues of $E$ are positive it is easy to see that $\left\langle x, t^{E} \theta\right\rangle=1$ for at most countably many $t>0$ and hence the inner integral on the right hand side of the equation above is zero for every $\theta \in S$ and hence $\phi\{y:\langle x, y\rangle=1\}=0$ for every $x \in \Gamma$.

Next we show that $\phi(\Gamma)=\infty$. Since $\phi(\Gamma) \supseteq \lim _{\delta \rightarrow 0} \phi\{x:|\langle x, \theta\rangle| \geq \delta\}$ all we have to show is that for some $\|\theta\|=1$

$$
\lim _{\delta \rightarrow 0} \phi\{y:|\langle y, \theta\rangle| \geq \delta\}=\infty .
$$

In view of (4.2) we have

$$
\phi\{y:|\langle y, \theta\rangle| \geq \delta\}=\frac{1}{s} \phi\left\{y:\left|\left\langle y, s^{E^{*}} \theta\right\rangle\right| \geq \delta\right\}
$$

for all $s>0$. Now write $s^{E^{*}} \theta=r_{s} \theta_{s}$ for some $\left\|\theta_{s}\right\|=1$ and $r_{s}>0$ and note that $r_{s}$ depends continuously on $s$ with $r_{s} \rightarrow 0$ as $s \rightarrow 0$ and $r_{s} \rightarrow \infty$ as $s \rightarrow \infty$. Choose $s=s(\delta)$ such that $r_{s}=\delta$. Then if $\delta \rightarrow 0$ we have $s=s(\delta) \rightarrow 0$. But then $\phi\{y:|\langle y, \theta\rangle| \geq \delta\}=(1 / s) \phi\left\{y:\left|\left\langle y, \theta_{s}\right\rangle\right| \geq 1\right\}$, so in view of (4.6) it remains to show that

$$
\inf _{\|\theta\|=1} \phi\{y:|\langle y, \theta\rangle| \geq 1\}>0 .
$$

For $\|\theta\|=1$ let $\rho(\theta)=\phi\{y:|\langle y, \theta\rangle| \geq 1\}$. Since $\rho(\theta) \leq \phi\{y:\|y\| \geq$ $1\}<\infty, \rho$ is finite for all $\|\theta\|=1$. We first show that $\rho$ is continuous. Let $\varepsilon>0$ be arbitrary and $\theta_{n} \rightarrow \theta$ where $\left\|\theta_{n}\right\|=1$ for all $n$. Since $\left.\phi\right|_{\{x:\|x\| \geq \delta\}}$ is a finite Borel measure for every $\delta>0$ there exists a $R>1$ such that for $D=\{x \in \mathbb{R}\urcorner:\|\curvearrowleft\| \geq \mathbb{R}\}$ we have $\phi(D)<\varepsilon / 4$. Then

$$
\begin{aligned}
\left|\rho\left(\theta_{n}\right)-\rho(\theta)\right| & =\left|\phi\left\{y:\left|\left\langle y, \theta_{n}\right\rangle\right| \geq 1\right\}-\phi\{y:|\langle y, \theta\rangle| \geq 1\}\right| \\
& \leq\left|\phi\left(\left\{y:\left|\left\langle y, \theta_{n}\right\rangle\right| \geq 1\right\} \cap D^{c}\right)-\phi\left(\{y:|\langle y, \theta\rangle| \geq 1\} \cap D^{c}\right)\right|+\frac{\varepsilon}{2} \\
& \leq \phi\left(\left(\left\{y:\left|\left\langle y, \theta_{n}\right\rangle\right| \geq 1\right\} \triangle\{y:|\langle y, \theta\rangle| \geq 1\}\right) \cap D^{c}\right)
\end{aligned}
$$

where $A \triangle B$ denotes the symmetric difference of the set $A, B \subset \Gamma$ and $A^{c}$ denotes the complement of a set $A$. A simple geometrical argument shows that

$\left(\left\{y:\left|\left\langle y, \theta_{n}\right\rangle\right| \geq 1\right\} \triangle\{y:|\langle y, \theta\rangle| \geq 1\}\right) \cap D^{c} \subset\left\{y:|\langle y, \theta\rangle| \in\left[1-\varepsilon_{n}, 1+\varepsilon_{n}\right]\right\} \cap D^{c}$ for some $\varepsilon_{n}>0$ depending on $\theta_{n}, \theta$ and $R$ with $\varepsilon_{n} \rightarrow 0$ as $n \rightarrow \infty$. But

$$
\phi\left(\left\{y:|\langle y, \theta\rangle| \in\left[1-\varepsilon_{n}, 1+\varepsilon_{n}\right]\right\} \cap D^{c}\right) \rightarrow \phi\left(\{y:|\langle y, \theta\rangle|=1\} \cap D^{c}\right) \rightarrow 0
$$

as $n \rightarrow \infty$ since we already know that $\phi\{y:\langle y, \theta\rangle=1\}=0$ for all $\theta \in \Gamma$. Hence $\rho$ is a continuous function. In order to prove (4.7) it now suffices to show that $\phi\{y:|\langle y, \theta\rangle| \geq 1\}>0$ for all $\|\theta\|=1$. If $\phi\left\{y:\left|\left\langle y, \theta_{0}\right\rangle\right| \geq 1\right\}=0$ for some $\left\|\theta_{0}\right\|=1$ it follows as in Remark 4.3 that $\phi$ is not full, which is a contradiction. A similar argument shows that $\phi\{y:|\langle y, x\rangle|>1\}>0$ for any $x \in \Gamma$, and this concludes the proof. 
Our next result shows that admissible $\phi \in \mathcal{M}$ always define an admissible function $\varphi \in \mathcal{F}$. For $x \in \Gamma$ let $H_{x}=\{y \in \Gamma:|\langle x, y\rangle|>1\}$ and note that for $\mu \in \mathcal{M}$ the function $F(x)=\mu\left(H_{x}\right)$ belongs to $\mathcal{F}$.

Theorem 4.8. Let $\phi \in \mathcal{M}$ be admissible. For $x \in \Gamma$ define $\varphi(x)=\phi\left(H_{x}\right)$. Then $\varphi \in \mathcal{F}$ is admissible.

Proof. We first show that $\varphi$ is continuous. Let $\varepsilon>0$ be arbitrary and assume that $x_{n} \rightarrow x$ in $\Gamma$. Since $\left.\phi\right|_{\{x:\|x\|>\delta\}}$ is a finite measure, for every $\delta>0$ there exists a $R>0$ such that for $D=\{x:\|x\| \geq R\}$ we have $\phi(D)<\varepsilon / 4$. Then

$$
\begin{aligned}
\left|\varphi\left(x_{n}\right)-\varphi(x)\right| & \leq\left|\phi\left(H_{x_{n}} \cap D^{c}\right)-\phi\left(H_{x} \cap D^{c}\right)\right|+\frac{\varepsilon}{2} \\
& =\phi\left(\left(H_{x_{n}} \triangle H_{x}\right) \cap D^{c}\right)+\frac{\varepsilon}{2} .
\end{aligned}
$$

By an argument similar to the proof of Theorem 4.7 there exist $\varepsilon_{n}>0$, $\varepsilon_{n} \rightarrow 0$ such that

$$
\left(H_{x_{n}} \triangle H_{x}\right) \cap D^{c} \subset\left\{y:|\langle x, y\rangle| \in\left[1-\varepsilon_{n}, 1+\varepsilon_{n}\right]\right\} \cap D^{c}
$$

and hence

$$
\begin{aligned}
\phi\left(\left(H_{x_{n}} \triangle H_{x}\right) \cap D^{c}\right) & \leq \phi\left(\left\{y:|\langle x, y\rangle| \in\left[1-\varepsilon_{n}, 1+\varepsilon_{n}\right]\right\} \cap D^{c}\right) \\
& \rightarrow \phi\left(\{y:|\langle x, y\rangle|=1\} \cap D^{c}\right)=0
\end{aligned}
$$

as $n \rightarrow \infty$ since $\phi\{y:|\langle x, y\rangle|=1\}=0$ by assumption, and it follows that $\varphi$ is continuous.

Assume now that $\left\|x_{t}\right\| \rightarrow \infty$ and note that $\varphi\left(x_{t}\right)=\phi\left\{y:\left|\left\langle x_{t}, y\right\rangle\right|>1\right\}$. Write $x_{t}=r_{t} \theta_{t}$ for some $r_{t}>0$ and $\left\|\theta_{t}\right\|=1$. If $\theta_{t} \rightarrow \theta_{0}$ along a subsequence then along that subsequence

$$
\begin{aligned}
\phi\left\{y:\left|\left\langle x_{t}, y\right\rangle\right|>1\right\} & =\phi\left\{y:\left|\left\langle\theta_{t}, y\right\rangle\right|>r_{t}^{-1}\right\} \\
& \rightarrow \phi\left\{y:\left|\left\langle\theta_{0}, y\right\rangle\right| \geq 0\right\}=\phi(\Gamma)=\infty .
\end{aligned}
$$

Since every subsequence contains a further subsequence with that property, we have $\varphi\left(x_{t}\right) \rightarrow \infty$ whenever $\left\|x_{t}\right\| \rightarrow \infty$ which concludes the proof.

Next we show that the convergence in $\mathcal{M}$ naturally leads to the convergence in $\mathcal{F}$.

Theorem 4.9. Let $\mu_{n} \in \mathcal{M}$, let $\phi \in \mathcal{M}$ be admissible and define $\varphi(x)=$ $\phi\left(H_{x}\right)$ and $F_{n}(x)=\mu_{n}\left(H_{x}\right)$. Then $\mu_{n} \rightarrow \phi$ in $\mathcal{M}$ implies $F_{n} \rightarrow \varphi$ in $\mathcal{F}$. 
Proof. Assume that $\mu_{n} \rightarrow \phi$ in $\mathcal{M}$. Given $\varepsilon>0$ choose $M>0$ large enough such that for $D=\{x:\|x\| \geq M\}$ we have $\phi(D)<\varepsilon / 2$. Since by Theorem $4.8 \varphi$ is admissible it is continuous and hence for any $x \in \Gamma$ we can choose a $\delta>0$ such that $\varphi((1-\delta) x) \leq \varphi(x)+\varepsilon / 2$. Let $x_{n} \rightarrow x$ in $\Gamma$. Then a simple geometrical argument shows that there exists a $n_{0} \geq 1$ such that for all $n \geq$ $n_{0}$ we have $H_{x_{n}} \subset H_{x(1-\delta)} \cup D$. Hence $F_{n}\left(x_{n}\right)=\mu_{n}\left(H_{x_{n}}\right) \leq \mu_{n}\left(H_{x(1-\delta)}\right)+$ $\mu_{n}(D)$, and $\mu_{n} \rightarrow \phi$ together with the Portemanteau theorem for finite measures implies that $\mu_{n}\left(H_{x(1-\delta)}\right) \rightarrow \phi\left(H_{x(1-\delta)}\right)$ since $\phi\left(\partial H_{x(1-\delta)}\right)=0$, and $\lim \sup _{n \rightarrow \infty} \mu_{n}(D) \leq \phi(D)$. Therefore

$$
\begin{aligned}
\limsup _{n \rightarrow \infty} F_{n}\left(x_{n}\right) & \leq \phi\left(H_{x(1-\delta)}\right)+\phi(D) \\
& \leq \varphi(x(1-\delta))+\frac{\varepsilon}{2} \\
& \leq \varphi(x)+\varepsilon .
\end{aligned}
$$

Since $\varepsilon>0$ is arbitrary we have shown that

$$
\limsup _{n \rightarrow \infty} F_{n}\left(x_{n}\right) \leq \varphi(x) .
$$

Next, using the fact that $\varphi$ is admissible again, we can choose a $\delta>0$ such that $\varphi((1+\delta) x) \geq \varphi(x)-\varepsilon / 2$ and it is easy to see that $H_{x_{n}} \supset H_{(1+\delta) x} \cap D^{c}$ for all large $n$ and hence $F_{n}\left(x_{n}\right) \geq \mu_{n}\left(H_{(1+\delta) x} \cap D^{c}\right)$. But $H_{(1+\delta) x} \cap D^{c}$ is open and therefore the Portemanteau theorem for finite measures implies that

$$
\begin{aligned}
\liminf _{n \rightarrow \infty} F_{n}\left(x_{n}\right) & \geq \phi\left(H_{(1+\delta) x} \cap D^{c}\right) \\
& \geq \phi\left(H_{(1+\delta) x}\right)-\frac{\varepsilon}{2} \\
& =\varphi((1+\delta) x)-\frac{\varepsilon}{2} \\
& \geq \varphi(x)-\varepsilon,
\end{aligned}
$$

so we also have $\liminf _{n \rightarrow \infty} F_{n}\left(x_{n}\right) \geq \varphi(x)$. This together with (4.8) concludes the proof.

Theorem 4.9 can be used to show that if $\mu$ is regularly varying then the function $F(x)=\mu\left(H_{x}\right)$ is also regularly varying. One might argue that if the transformation $F(x)=\mu\left(H_{x}\right)$ from measures to functions would be one-to-one the theory of regularly varying measures could be obtained from the results for functions. If $\mu_{1}, \mu_{2} \in \mathcal{M}$ are finite measures then if $F_{1}(x)=\mu_{1}\left(H_{x}\right)=\mu_{2}\left(H_{x}\right)=F_{2}(x)$ for all $x \in \Gamma$ it follows from the uniqueness of the Fourier transform for finite measures that $\mu_{1}=\mu_{2}$. But if the measures involved have a singularity at the origin this in general is no longer the case as shown in the following example. 
Example 4.10. On $\mathbb{R}^{\not \models}$ we define for Borel subsets $A \subset \Gamma$

$$
\phi(A)=\int_{S^{1}} \int_{0}^{\infty} 1_{A}(r u) \frac{d r}{r^{2}} d \lambda(u)
$$

where $\lambda$ is a finite Borel measure on the unit sphere $S^{1}$. This is the general form of the Lévy measure of a multivariate 1-stable distribution on $\mathbb{R}^{\not z}$, see [19]. For $\theta \in S^{1}$ and $t>0$ compute that

$$
\phi\{y:\langle y, \theta\rangle>t\}=\frac{1}{t} G(\theta)
$$

where

$$
G(\theta)=\int_{S^{1}}\langle u, \theta\rangle 1_{\{u:\langle u, \theta\rangle>0\}}(u) d \lambda(u) .
$$

Now let $\lambda_{1}$ be the Lebesque measure on $S^{1}$ with $\lambda_{1}\left(S^{1}\right)=2 \pi$. Since $\lambda_{1}$ is rotation invariant it follows that the corresponding function $G_{1}$ defined by (4.11) is constant, say $G_{1}(\theta)=D>0$ for all $\theta \in S^{1}$ and some constant $D$. Now let

$$
C=\bigcup_{l=0}^{2}\left\{(\cos t, \sin t): \frac{2 l \pi}{3} \leq t<\frac{(2 l+1) \pi}{3}\right\}
$$

and define $\lambda_{2}(A)=M \lambda_{1}(A \cap C)$ for some $M>0$ and let $G_{2}$ be the corresponding function defined by (4.11). Then an elementary computation shows that $G_{2}(\theta)=c M$ for some constant $c>0$ and all $\theta \in S^{1}$. Hence an appropriate choice of $M$ yields $G_{1}(\theta)=G_{2}(\theta)$ for all $\theta \in S^{1}$. Now let $\phi_{1}$ and $\phi_{2}$ be defined by (4.9) with $\lambda_{1}$ and $\lambda_{2}$, respectively. In view of (4.10) we have $\phi_{1}\left(H_{x}\right)=\phi_{1}\{y:|\langle y, x /\|x\|\rangle|>1 /\|x\|\}=\phi_{2}\left(H_{x}\right)$ for all $x \in \Gamma$, but since $\operatorname{supp} \phi_{1} \neq \operatorname{supp} \phi_{2}$, where supp denotes the support, we have $\phi_{1} \neq \phi_{2}$.

Now we will derive our theory of multivariate regularly varying measures. The results and proofs are quite similar to the function case considered above.

Definition 4.11. An operator $A \in G L\left(\mathbb{R}^{\urcorner}\right)$is called a symmetry of a measure $\phi \in \mathcal{M}$ if $\phi\{A d x\}=\phi\{d x\}$. Let

$$
\mathcal{S}(\phi)=\left\{A \in G L\left(\mathbb{R}^{\urcorner}\right): \mathbb{A} \text { ia a symmetry of } \phi\right\} .
$$

For a linear operator $A$ on $\mathbb{R}^{\urcorner}$let $A^{*}$ denote the transpose of $A$.

Lemma 4.12. Let $\phi \in \mathcal{M}$ and define $\varphi(x)=\phi\left(H_{x}\right)$. Then $\mathcal{S}(\phi) \subset\{A \in$ $\left.G L\left(\mathbb{R}^{\urcorner}\right):\left(\mathbb{A}^{*}\right)^{-\nVdash} \in \mathcal{S}(\varphi)\right\}$.

Proof. Let $A \in \mathcal{S}(\phi)$ and note that $A H_{x}=H_{\left(A^{*}\right)^{-1} x}$. Then $\varphi(x)=$ $\phi\left(H_{x}\right)=\phi\left(A H_{x}\right)=\phi\left(H_{\left(A^{*}\right)^{-1} x}\right)=\varphi\left(\left(A^{*}\right)^{-1} x\right)$ for all $x \in \Gamma$ showing that $\left(A^{*}\right)^{-1} \in \mathcal{S}(\varphi)$. 
Theorem 4.13. The symmetries $\mathcal{S}(\phi)$ of an admissible measure $\phi \in \mathcal{M}$ form a compact subgroup of $G L(\mathbb{R}\urcorner)$.

Proof. Trivially $\mathcal{S}(\phi)$ is a group. Show using similar arguments as in the proof of Theorem 3.5 to show that $\mathcal{S}(\phi)$ is closed. Let $\varphi(x)=\phi\left(H_{x}\right)$. By Theorem 4.8, $\varphi$ is admissible and hence $\mathcal{S}(\varphi)$ is a compact subgroup of $G L(\mathbb{R}\urcorner)$ using Theorem 3.5. An application of Lemma 4.12 concludes the proof.

Now let us define $A \mu\{d x\}=\mu\left\{A^{-1} d x\right\}$ for any $\mu \in \mathcal{M}$ and any $A \in$ $G L(\mathbb{R}\urcorner)$.

Theorem 4.14 (Convergence of types). Let $\mu_{n} \in \mathcal{M}$, let $\phi, \phi_{0} \in \mathcal{M}$ be admissible and $\left.A_{n}, B_{n} \in G L(\mathbb{R}\urcorner\right)$. Suppose that $B_{n} \mu_{n} \rightarrow \phi$. Then $A_{n} \mu_{n} \rightarrow$ $\phi_{0}$ if and only if $\phi_{0}=C \phi$ and $A_{n} B_{n}^{-1} \rightarrow C \mathcal{S}(\phi)$ for some $C \in G L\left(\mathbb{R}^{\urcorner}\right)$.

Proof. If $A_{n} \mu_{n} \rightarrow \phi_{0}$ then $\left(A_{n} B_{n}^{-1}\right)\left(B_{n} \mu_{n}\right) \rightarrow \phi_{0}$. Then by an argument similar to the proof of Lemma 3.8 it follows that $\phi_{0}=C \phi$ for some $C \in$ $G L\left(\mathbb{R}^{\urcorner}\right)$. Then $C^{-1}\left(A_{n} B_{n}^{-1}\right)\left(B_{n} \mu_{n}\right) \rightarrow \phi$ and then using the same kind of arguments as in the proof of Lemma 3.7 we get $C^{-1}\left(A_{n} B_{n}^{-1}\right) \rightarrow \mathcal{S}(\phi)$. Multiply both sides by $C$ on the left. Conversely if $A_{n} B_{n}^{-1} \rightarrow C \mathcal{S}(\phi)$ then since $\mathcal{S}(\phi)$ is compact by Theorem 4.13 any subsequence contains a further subsequence $\left(n^{\prime}\right)$ such that $A_{n} B_{n}^{-1} \rightarrow C G$ for some $G \in \mathcal{S}(\phi)$. Hence $A_{n} \mu_{n}=\left(A_{n} B_{n}^{-1}\right)\left(B_{n} \mu_{n}\right) \rightarrow(C G) \phi=C \phi=\phi_{0}$ along $\left(n^{\prime}\right)$. This concludes the proof.

Theorem 4.15. Let $\mu \in \mathcal{M}, \phi \in \mathcal{M}$ admissible and $A_{n} \in G L\left(\mathbb{R}^{\urcorner}\right)$. Suppose $a_{n}>0$ is a regularly varying sequence with index $\beta \neq 0$ and that $a_{n}\left(A_{n} \mu\right) \rightarrow \phi$. Then there exists a linear operator B such that for all $t>0$ : $t^{\beta} \cdot \phi=t^{B} \phi$ and

$$
A_{[t n]} A_{n}^{-1} \rightarrow t^{-B} \mathcal{S}(\phi)
$$

as $n \rightarrow \infty$.

Proof. We argue as in the proof of Theorem 3.10. Let $\mu_{n}=a_{n} \cdot \mu$ so that $A_{n} \mu_{n} \rightarrow \phi$. Then $A_{[t n]} \mu_{n} \rightarrow t^{-\beta} \cdot \phi$ for any $t>0$. Theorem 4.14 yields $t^{-\beta} \cdot \phi=C_{t} \phi$ and $A_{[t n]} A_{n}^{-1} \rightarrow C_{t} \mathcal{S}(\phi)$ for all $t>0$ and some $C_{t} \in G L\left(\mathbb{R}^{\urcorner}\right)$. Define $G_{t}=C_{t} \mathcal{S}(\phi), G=\bigcup_{t>0} G_{t}$ and show that $G$ is a closed subgroup of $G L\left(\mathbb{R}^{\urcorner}\right)$. Define $\zeta: G \rightarrow \mathbb{R}^{+}, \zeta(C)=t$ for all $C \in G_{t}$ to obtain a continuous group homomorphism. It follows as before that there exists a linear operator $B$ such that $t^{-B} \in G_{t}$ for all $t>0$ and so we may write $G_{t}=t^{-B} \mathcal{S}(\phi)$ which concludes the proof. 
In case of Theorem 4.15, if we define $E=\beta^{-1} B$ then (4.2) holds for all $t>0$. In other words we have $t \cdot \phi=t^{E} \phi$ for all $t>0$.

Definition 4.16. Let $\phi \in \mathcal{M}$. Then any linear operator $E$ such that

$$
t \cdot \phi=t^{E} \phi
$$

holds for all $t>0$ is called an exponent of $\phi$. Let

$$
\mathcal{E}(\phi)=\{E: E \text { is an exponent of } \phi\} .
$$

Theorem 4.17. Let $\phi \in \mathcal{M}$ be admissible. Then the exponents and symmetries of $\phi$ are related by

$$
\mathcal{E}(\phi)=E+T \mathcal{S}(\phi)
$$

where $E \in \mathcal{E}(\phi)$ is arbitrary and $T \mathcal{S}(\phi)$ denotes the tangent space. One can always select an exponent $E_{0}$ which commutes with every symmetry $G \in \mathcal{S}(\phi)$.

Proof. We argue as in the proof of Theorem 3.12. Let $G$ and $\zeta$ be as in the proof of Theorem 4.15. Define a continuous group homomorphism $L: T G \rightarrow \mathbb{R}$ by letting $L(X)=\log \zeta(\exp (X))$, where exp $: T G \rightarrow G$ is the exponential mapping. Then $T \mathcal{S}(\phi)=\operatorname{ker} L$ and $E \in \mathcal{E}(\phi)$ if and only if $E \in T G$ and $L(E)=-1 / \beta$. Since $L$ is a linear functional $\mathcal{E}(\phi)=E+T \mathcal{S}(\phi)$ follows. Then the existence of a commuting exponent follows exactly as in the proof of Theorem 3.12.

If $B$ is the linear operator in Theorem 4.15 then we know that $E=\beta^{-1} B$ is an element of $\mathcal{E}(\phi)$. Suppose $E^{\prime} \in \mathcal{E}(\phi)$ is another exponent and let $B^{\prime}=\beta E^{\prime}$. Then Theorem 4.17 implies that $E^{\prime}=E+X$ where $X \in T \mathcal{S}(\phi)$. Using the fact that $t^{X} \in \mathcal{S}(\phi)$, it is easy to see that $t^{-B^{\prime}} \mathcal{S}(\phi)=t^{-B} \mathcal{S}(\phi)$ for all $t>0$. Then we may assume that the linear operator $B$ in (4.12) commutes with every symmetry.

Theorem 4.18. Let $\mu \in \mathcal{M}$ and $\phi \in \mathcal{M}$ be admissible and $A_{n} \in G L\left(\mathbb{R}^{\urcorner}\right)$. Suppose $a_{n}>0$ is a regularly varying sequence with index $\beta \neq 0$ and that $a_{n}\left(A_{n} \mu\right) \rightarrow \phi$. Then $\mu$ is regularly varying with index $E$ for any $E \in \mathcal{E}(\phi)$.

Proof. The proof is similar to the proof of Theorem 3.14. Theorem 4.15 implies that (4.12) holds for some linear operator $B$ and Lemma 2.2 shows that this convergence is uniform on compact subsets of $t>0$. We may take $B=\beta E$ where $E \in \mathcal{E}(\phi)$ is arbitrary. To begin with we will assume that $E$ is commuting so that $t^{-B} G=G t^{-B}$ for all $t>0$ and all $G \in \mathcal{S}(\phi)$. Now suppose that $G_{n} \in \mathcal{S}(\phi)$ and let $B_{n}=A_{n} G_{n}$. Since by Theorem $4.13 \mathcal{S}(\phi)$ 
is compact it follows that $a_{n}\left(B_{n} \mu\right) \rightarrow \phi$ too. Now the proof follows along the same lines as the proof of Theorem 3.14.

\section{Remarks}

Some of the results in this paper extend the work of probabilists interested in the behavior of random vectors and probability distributions on $\mathbb{R}\urcorner$. The convergence of types theorem for probability measures on $\mathbb{R}\urcorner$ is due to Fisz [7] and Billingsley [2]. The limit measure $\phi$ in (4.1) appears in a natural way as a kind of spectral measure in the Fourier transform of a class of probability measures called operator-stable laws, which were introduced by Sharpe [21]. While the methods of this paper can be used to give a simpler proof of Sharpe's results, our proof of Theorem 3.10 was inspired by Sharpe's work. The subsequent work of Holmes, Hudson, and Mason [10] and Hudson, Jurek, and Veeh [11] is reflected in Theorem 3.12. The spectral decomposition theorem presented here extends a result of the authors which appeared in [15] and [17]. Theorem 3.14 above extends a recent result from [16]. The multivariable theory of regularly varying functions and measures was generalized recently to the class of R-O varying measures which form a broader class appropriate for investigations of operator semistable laws and their generalized domains of attraction as well as stochastic compactness results for R-O varying measures, see [18].

Acknowledgement. We thank the anonymous referee for a number of insightful comments, especially for pointing out an error in an earlier version of the paper.

\section{References}

[1] Balkema, A.A., Monotone Transformations and Limit Laws, Mathematical Centre Tracts 45, Mathematisch Centrum, Amsterdam, 1973.

[2] Billingsley, P., Convergence of types in k-space, Z. Wahrsch. verw. Geb. 5 (1966), 175-179.

[3] Billingsley, P., Convergence of Probability Measures, Wiley, New York, 1968.

[4] Bingham, N., Goldie, C. and Teugels, J., Regular Variation, Encyclopedia of Mathematics and its Applications 27, Cambridge University Press, Cambridge, 1987.

[5] Curtis, C., Linear Algebra, 3rd ed., Allyn and Bacon, Boston, 1974.

[6] Feller, W., An Introduction to Probability Theory and Its Applications, Vol. II, 2nd Ed., Wiley, New York, 1971.

[7] Fisz, M., A generalization of a theorem of Khintchin, Studia Math. 14 (1954), 310313.

[8] de Haan, L., Omey, E. and Resnick, S., Domains of attraction and regular variation in $\mathbb{R}$, J. Multivatiate Anal. 14 (1984), 17-33. 
[9] Hirsch, M. and Smale, S., Differential Equations, Dynamical Systems and Linear Algebra, Academic Press, New York, 1974.

[10] Holmes, J., Hudson, W. and Mason, J.D., Operator stable laws: multiple exponents and elliptical symmetry, Ann. Probab. 10 (1982), 602-612.

[11] Hudson, W., Jurek, Z. and Veeh, J., The symmetry group and exponents of operator stable probability measures Ann. Probab. 14 (1986), 1014-1023.

[12] Jakimiv, A.L., Many-dimensional Tauberian Theorems and their application to Bellman-Harris branching processes (in Russian), Math. Sbornik 115(157) (1981), 463-477.

[13] Jurek, Z.J., Polar coordinates in Banach spaces, Bull. Acad. Pol. Math. 32 (1984), 61-66.

[14] Meerschaert, M., Regular variation in $\mathbb{R}^{\urcorner}$, Proc. Amer. Math. Soc. 102 (1988), 341348.

[15] Meerschaert, M., Spectral decomposition for generalized domains of attraction, Ann. Probab. 19 (1991), 875-892.

[16] Meerschaert, M., Norming operators for generalized domains of attraction, J. Theoret. Probab. 7 (1994), 793-798.

[17] Meerschaert, M. and Scheffler, H.-P., Spectral decomposition for generalized domains of semistable attraction, J. Theoret. Probab. 10 (1997), 51-71.

[18] Scheffler, H.-P., Multivariable $R$-O variation and generalized domains of semistable attraction, Habilitation Thesis, University of Dortmund, 1997.

[19] Samorodnitsky, G. and Taqqu, M.S., Stable non-Gaussian Random Processes, Chapman and Hall, New York, 1994.

[20] Seneta, E., Regularly Varying Functions, Lecture Notes in Math. 508, Springer, Berlin, 1976.

[21] Sharpe, M., Operator-stable probability distributions on vector groups, Trans. Amer. Math. Soc. 136 (1969), 51-65.

[22] Stam, A., Regular Variation in $\mathbb{R}_{+}$and the Abel-Tauber Theorem, Technical Report T. W. 189, Mathematisch Instituut Rijksuniversiteit Groningen, The Netherlands, 1977.

Mark M. MeERschaert

DEPARTMENT OF MATHEMATICS

UNIVERSITY OF NEVADA

RENO NV 89557

USA
HANS-Peter Scheffler

DEPARTMENT OF MATHEMATICS

UNIVERSITY OF DORTMUND 44221 DORTMUND GERMANY 